\title{
Expression of keratinocyte growth factor receptor correlates with expansive growth and early stage of gastric cancer
}

\author{
TETSURO MATSUNOBU ${ }^{1,3}$, TOSHIYUKI ISHIWATA $^{1}$, MASANORI YOSHINO $^{1,3}$, MASANORI WATANABE $^{3}$, \\ MITSUHIRO KUDO $^{1}$, KOSHI MATSUMOTO $^{4}$, AKIRA TOKUNAGA ${ }^{3}$, TAKASHI TAJIRI $^{2}$ and ZENYA NAITO $^{1}$ \\ ${ }^{1}$ Department of Integrative Pathology, Graduate School of Medicine (Department of Pathology II), \\ ${ }^{2}$ Surgery for Organ and Biological Regulation, Graduate School of Medicine (Department of Surgery I), \\ 1-1-5 Sendagi, Bunkyo-ku, Tokyo 113-8602; ${ }^{3}$ Center for Digestive Diseases and ${ }^{4}$ Division of Pathology, \\ Second Hospital of Nippon Medical School, 1-396 Kosugicho, Nakahara-ku, Kawasaki, Kanagawa 211-8533, Japan
}

Received September 9, 2005; Accepted October 28, 2005

\begin{abstract}
The keratinocyte growth factor receptor, also known as KGFR/FGFR2 IIIb, is mainly localized in epithelial cells, and participates in the proliferation of these cells. In contrast, a recent study has revealed that the overexpression of KGFR in salivary adenocarcinoma induces growth inhibition, cell differentiation and apoptosis. We attempted to clarify the expression and role of KGFR in normal and cancerous human gastric tissues and cancer cell lines. Reverse-transcription polymerase chain reaction and Western blot analyses showed KGFR mRNA and its protein expression in NUGC-4, KATOIII and MKN-7 gastric cancer cell lines, but not in the NS-8 cell line. Immunohistochemically, KGFR immunoreactivity was weakly detected in the luminal surface of normal gastric epithelial cells. In addition, KGFR immunoreactivity was strongly detected in the nucleus and cytoplasm of many parietal cells. In gastric cancer tissue, KGFR was expressed in the cell membrane and cytoplasm of cancer cells in 46 of 126 (36.5\%) cases. KGFR expression in gastric cancer cells was significantly associated with early-type macroscopic findings, shallow invasion of the gastric wall and expansive growth type. KGFR expression tended to correlate with a good prognosis in gastric cancer. These findings indicate that KGFR expression plays important roles in the differentiation of normal gastric epithelial cells and parietal cell functions. Furthermore, a decreased expression level or the non-expression of KGFR in gastric cancer cells may be associated with the proliferation and invasion of gastric cancer cells and a poor prognosis for the patient.
\end{abstract}

Correspondence to: Dr Zenya Naito, Department of Integrative Pathology, Graduate School of Medicine, Nippon Medical School, 1-1-5 Sendagi, Bunkyo-ku, Tokyo 113-8602, Japan

E-mail: naito@nms.ac.jp

Key words: keratinocyte growth factor receptor, gastric cancer, KGF/FGF-7, immunohistochemistry

\section{Introduction}

Gastric cancer is a neoplastic disease with a high incidence and is one of the leading causes of death worldwide, particularly in Asia. The expressions of various growth factors and their receptors in gastric cancer cells have been reported, including epidermal growth factor (EGF), transforming growth factor (TGF)- $\alpha$, TGF- $\beta$, hepatocyte growth factor (HGF), platelet derived growth factor (PDGF) and vascular endothelial growth factor (VEGF) (1-4).

Fibroblast growth factors (FGFs) 1-23 have been identified in humans and mice. FGF-19 is the human ortholog of mouse Fgf15. Thus, the FGF family contains at least 22 members with molecular masses ranging from 17 to $34 \mathrm{kDa}$ and share a $13-71 \%$ amino acid identity $(5,6)$. The biological activities of FGFs are mediated by a family of type I transmembrane tyrosine kinase receptors, FGF receptors (FGFRs) that undergo dimerization and autophosphorylation after ligand binding. There are four FGFRs (FGFRs 1-4); FGFRs 1-3 have two isoforms named IIIb and IIIc $(7,8)$. FGFR-2 IIIb is known as the keratinocyte growth factor receptor (KGFR). The $\mathrm{Kgfr}$ gene is known to be included in the category of $K$-sam, Fgfr 2 and bek genes. KGFR is the product of an alternative splice variant of the $F g f r 2$ gene (7). KGFR is specifically expressed in epithelial cells, and FGF-1, FGF-3, FGF-4, FGF-6, the keratinocyte growth factor (KGF/FGF-7) and FGF-10 (KGF-2) have a high affinity to $\operatorname{KGFR}(8,9)$.

Mice without the Fgfr $2 I I I b$ isoform, but with retaining Fgfr2 IIIc, show dysgeneses of the kidneys, salivary glands, adrenal glands, thymus, pancreas, skin, otic vesicles, hair follicles and glandular stomach, and agenesis of the lungs, anterior pituitary glands, thyroid glands, teeth and limbs (10-12). KGFR is expressed in epithelial cells throughout the gastrointestinal tract, and an intraperitoneal injection of KGF enhances normal intestinal epithelial growth $(13,14)$. Regarding cancerous tissue, KGFR expression was previously reported in pancreatic cancer and colorectal cancer cells $(15,16)$. Furthermore, pancreatic cancer cell line growth was stimulated by recombinant KGF administration (17), and dose-dependent cell growth by KGF was observed in welldifferentiated colorectal cancer cell lines (18). On the other 
hand, a stable transfectant of the Kgfr gene of salivary adenocarcinoma cells induced cancer cell differentiation and apoptosis (19). These lines of evidence suggest that KGFR plays important roles in the regulation of epithelial cell and cancer cell functions, such as growth, differentiation and apoptosis.

FGFRs have been implicated in the tumorigenesis of a wide range of cancers, including cancers of the pancreas, breast and stomach and glioblastoma multiforme. K-sam overexpression is associated with the malignant behavior of an undifferentiated type of stomach cancer, such as infiltrative growth and metastasis. Macroscopically, Borrmann type IV or diffusely infiltrative stomach cancer cells are frequently stained for K-sam compared with other types of gastric cancer cell $(20,21)$. Gastric fibroblasts specifically stimulate the growth of scirrhous gastric cancer cells but not that of welldifferentiated adenocarcinoma cells. Fibroblasts, particularly gastric fibroblasts, express KGF mRNA, whereas gastric cancer cells do not $(22,23)$. Conversely, scirrhous gastric cancer cells more strongly express KGFR mRNA than well-differentiated gastric adenocarcinoma cells, whereas gastric fibroblasts do not express KGFR mRNA. By immunohistochemical analysis, many undifferentiated types of advanced stomach cancer have been shown to be K-sam-positive, whereas no cases of the K-sam-positive differentiated or intestinal type have been revealed (21). The co-inoculation of scirrhous gastric cancer cells with gastric fibroblasts into nude mice specifically enhances tumorigenicity, compared with that of gastric cancer cells alone $(23,24)$. Furthermore, the histopathological findings of the xenograft produced by co-inoculation with gastric fibroblasts are similar to those of human scirrhous gastric carcinoma (24). All these lines of evidence suggest that KGF, synthesized by stromal fibroblasts and KGFR in cancer cells, has important roles in gastric cancer cell growth, particularly in undifferentiated types of cancer.

To date, the expression of KGFR in normal gastric epithelial cells and the role of KGFR in gastric cancer cells have not yet been completely clarified. In this study, we examined the localization of KGFR in normal gastric epithelial cells and correlation of KGFR expression in gastric cancer cells and the clinicopathological factors.

\section{Materials and methods}

Materials. The following were purchased: Histofine Simple Stain Max PO (R) or (M) kits and Simple Stain AP (R) kit from Nichirei (Tokyo, Japan); Immobilon PVDF membrane from Millipore (Yonezawa, Japan); M-PER Mammalian Protein Extraction reagent, a BCA protein assay kit and Super Signal West Pico Chemiluminescent substrates from Pierce (Rockford, IL, USA); horseradish peroxidase (HRP)conjugated anti-rabbit IgG antibody from Santa Cruz (Santa Cruz, CA, USA); Isogen from Nippon Gene (Tokyo, Japan); Takara RNA PCR kit (AMV) ver. 3.0 from Takara Biotech (Tokyo, Japan); superfrosted slides with a MAS coat from Matsunami Glass Ind., Ltd. (Osaka, Japan); Vectashield mounting medium containing 4', 6-diamidino-2-phenylindole dihydrochloride (DAPI) and fluorescein 5-isothiocyanate (FITC)-conjugated anti-rabbit IgG from Vector Laboratories, Inc. (Burlingame, CA, USA); malinol mounting medium from Mutoh Chemical Co. (Tokyo, Japan). All other chemicals and reagents were purchased from Sigma Chemical Corp. (St. Louis, MO, USA).

Human gastric cancer cell lines. NUGC-4, KATO-III, NS-8, and MKN-7 cell lines were obtained from the Cell Resource Center for Biomedical Research, Institute of Development, Aging and Cancer, Tohoku University (Sendai, Japan). The cells were grown in RPMI-1640 medium containing 10\% heat-inactivated fetal bovine serum (FBS), $100 \mathrm{U} / \mathrm{ml}$ penicillin, $100 \mu \mathrm{g} / \mathrm{ml}$ streptomycin and $0.25 \mu \mathrm{g} / \mu \mathrm{l}$ amphotericin B at $37^{\circ} \mathrm{C}$ under a humidified $5 \% \mathrm{CO}_{2}$ atmosphere.

Human gastric cancer tissue. Gastric carcinoma tissue samples were obtained from 126 patients (46 female and 80 male) during gastrectomy for gastric cancer at the Center for Digestive Diseases, Second Hospital of Nippon Medical School from 1994 to 1999 . The mean age of the cancer patients was 64.4 years (range 26-89 years). According to the criteria of the Japanese Classification of Gastric Carcinoma, there were 54 type 0,3 type 1,23 type 2, 28 type 3 and 18 type 4 gastric cancers (25). Histopathologically, there were 29 welldifferentiated adenocarcinomas, 29 moderately differentiated adenocarcinomas, 18 poorly differentiated adenocarcinomas (solid type), 19 poorly differentiated adenocarcinomas (nonsolid type), 25 signet-ring cell carcinomas, 4 mucinous adenocarcinomas and 2 papillary adenocarcinomas (Table I). Normal gastric tissue samples were also obtained from the surgical margin of the same patient. The tissues were fixed in $10 \%$ paraformaldehyde solution (PFA) for 18-20 h and then embedded in paraffin. All experimental procedures were approved by the Human Ethics Committee of the Second Hospital of Nippon Medical School.

Reverse-transcription polymerase chain reaction ( $R T-P C R)$. Total RNA extraction was performed using Isogen according to the manufacturer's protocol. Then, cDNA synthesis and PCR were performed using a Takara RNA PCR kit. The primers used for RT-PCR corresponded to nucleotides (nt) 1198-1217 (5'-CAC-TCG-GGG-ATA-AAT-AGT-TC-3') and nt 1330-1347 (5'-CGC-TTG-CTG-TTT-TGG-CAG-3') of the human KGFR cDNA (150 bp, accession no. U11814). The primer pair used for $B$-actin was (5'-TAC-ATG-GCTGGG-GTG-TTG-AA-3') and (5'-AAG-AGA-GGC-ATCCTC-ACC-CT-3') (218 bp). PCR was carried out using a Takara PCR thermal cycler MP (TP3300, Takara) for 30 cycles, each consisting of $1 \mathrm{~min}$ at $95^{\circ} \mathrm{C}, 1 \mathrm{~min}$ at $55^{\circ} \mathrm{C}$ and $2 \mathrm{~min}$ at $72^{\circ} \mathrm{C}$. The PCR products were analyzed using $2 \%$ agarose gel electrophoresis and direct sequencing was performed to confirm the sequences. Total RNA that was not subjected to reverse transcription was employed as the negative control.

Western blot analysis. The anti-KGFR antibody used was an affinity-purified rabbit polyclonal antibody raised against a peptide corresponding to amino acids of the human KGFR protein as previously reported (26). The proteins from the gastric cancer cell lines were subjected to sodium dodecyl sulfate polyacrylamide gel electrophoresis (SDS-PAGE) under reducing conditions and the separated proteins were transferred to Immobilon PVDF transfer membranes, which were then incubated for $16 \mathrm{~h}$ with the anti-KGFR antibody. 
Table I. Clinicopathological factors.

\begin{tabular}{l} 
Total \\
\hline Gender \\
Female \\
Male \\
Age (years) \\
$<50$ \\
$51-60$ \\
$61-70$ \\
$>71$
\end{tabular}

126

Macroscopic findings

0

1

2

3

4

Tumor size (cm)

$$
\begin{aligned}
& <2 \\
& 2-5 \\
& 5-10 \\
& \geq 10
\end{aligned}
$$

Histopathological type

Well-differentiated adenocarcinoma

Moderately differentiated adenocarcinoma

Poorly differentiated adenocarcinoma (solid)

Poorly differentiated adenocarcinoma (non-solid)

Signet-ring cell carcinoma

Mucinous adenocarcinoma

Papillary adenocarcinoma

Depth

$\mathrm{m}$
$\mathrm{sm}$
$\mathrm{mp}$
$\mathrm{ss}$
$\mathrm{se}$
$\mathrm{si}$

Stromal reaction

Medullary type

Intermediate type

Scirrhous type

Avaluative due to shallow invasion depth ${ }^{\mathrm{a}}$

Growth pattern

Expansive type

Intermediate type

Infiltrative type

Lymphatic invasion

Negative

Positive

Venous invasion

Negative

Positive
Table I. Continued.

Total

126

Regional lymph node metastasis

Negative

Positive

Stage

IA

IB

II

IIIA

IIIB

IV

${ }^{\mathrm{a} A c c o r d i n g}$ to Japanese Classification of Gastric Carcinoma (25).

The membranes were sequentially washed and incubated with a secondary HRP-conjugated anti-rabbit $\operatorname{IgG}$ antibody for $60 \mathrm{~min}$. After washing, the blots were visualized by enhanced chemiluminescence. To confirm the specificity of the positive band, prior to Western blot analysis, anti-KGFR antibody was preincubated with a blocking peptide of KGFR, and then subjected to Western blot analysis using the same protocol as described above.

Immunohistochemistry. The same anti-KGFR antibody used for the Western blot analysis was utilized for immunohistochemistry. Paraffin-embedded sections $(3 \mu \mathrm{m})$ were subjected to immunostaining using the Histofine Simple Stain Max PO (R) kit for KGFR. Then, endogenous peroxidase activity was blocked by incubation in $0.3 \%$ hydrogen peroxide in methanol for $30 \mathrm{~min}$. The tissue sections were incubated with the anti-KGFR (1:500 in dilution) antibody in phosphatebuffered saline (PBS) containing $1 \%$ bovine serum albumin (BSA) for $16 \mathrm{~h}$ at $4^{\circ} \mathrm{C}$. The bound antibodies were detected using the Simple Stain Max PO (R) reagent with diaminobenzidine tetrahydrochloride as the substrate. The sections were counter-stained with Mayer's hematoxylin. In some tissue sections, the bound antibodies were detected using the Simple Stain AP (R) reagent with New Fuchsin as the substrate. Counterstaining was not performed in these sections. For Ki-67 immunostaining, the tissue sections were preheated in $10 \mathrm{mM}$ citrate buffer ( $\mathrm{pH} 6.0$ ) for $15 \mathrm{~min}$ at $121^{\circ} \mathrm{C}$ in an autoclave oven. Then, endogenous peroxidase activity was blocked by incubation in $0.3 \%$ hydrogen peroxide in methanol for $30 \mathrm{~min}$. The tissue sections were incubated with an anti-Ki-67 (1:100 in dilution) antibody in PBS containing $1 \%$ BSA for $16 \mathrm{~h}$ at $4^{\circ} \mathrm{C}$. The bound antibodies were detected using the Simple Stain Max PO (M) reagent with diaminobenzidine tetrahydrochloride as the substrate. The sections were slightly counterstained with Mayer's hematoxylin.

Immunofluorescence staining and confocal laser microscopy. The same anti-KGFR antibody used for the Western blot analysis and immunohistochemistry was utilized for immunofluorescence staining. The tissue sections were incubated 


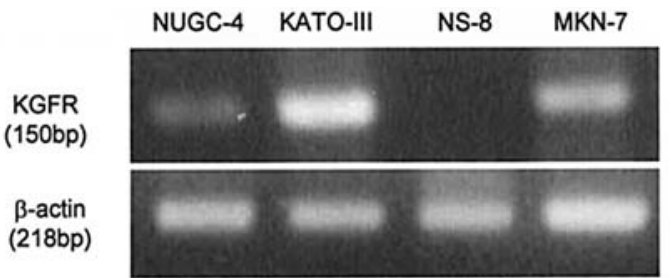

Figure 1. RT-PCR analysis of KGFR mRNA expression in gastric cancer cell lines. Total RNA was extracted from NUGC-4, KATO-III, NS-8 and MKN-7 gastric cell lines, and then cDNA synthesis and PCR were performed. KGFR mRNA at 150 bp was detected in NUGC-4, KATO-III and MKN-7 gastric cell lines (upper panel). B-actin mRNA at 218 bp was clearly detected in all cell lines (lower panel).

with the anti-KGFR (1:100 in dilution) antibody in PBS containing $1 \% \mathrm{BSA}$ for $16 \mathrm{~h}$ at $4^{\circ} \mathrm{C}$. Tissue sections were washed with PBS and then incubated with FITC-conjugated anti-rabbit IgG. One hour after incubation, the tissue sections were washed with PBS, and then mounted with Vectashield mounting medium containing DAPI. Fluorescent images were acquired using a confocal laser scanning microscope Digital Eclipse TE 2000-E (Nikon Insteck Co., Ltd. Tokyo, Japan) and a 100x immersion lens (Nikon Palm Apo VC) with blue diode (BD) and argon (Ar) lasers and were analyzed using the confocal microscope Digital Eclipse C1 control software EZ-C1 (version 2.30) (Nikon Insteck). The excitation wavelength for FITC was $488 \mathrm{~nm}$, and emission was selected and recorded using a 500- to 530-nm band-pass filter. In addition, the excitation wavelength for DAPI was $405 \mathrm{~nm}$, and emission was selected and recorded using a 432.5- to 4467.5-nm bandpass filter.

Assessment of KGFR immunostaining in cancer tissue. KGFR immunoreactivity in each tissue section was assessed by two investigators (Tetsuro Matsunobu and Toshiyuki Ishiwata) blind to the clinicopathological features of the tumor or patient survival. Positivity was initially classified according to the percentage of positive tumor cells regardless of staining intensity as follows: positive samples with no tumor cells or positive samples with less than $10 \%$ tumor cells were defined as negative, and positive samples with more than $10 \%$ cells were defined as positive.

Statistical analysis. Statistical significance was determined using the $\chi^{2}$ test. Survival rates were calculated for the 126 patients by using the Kaplan-Meier method. The log-rank test was used for univariate analysis to determine differences between curves. Multivariate analysis by Cox proportional hazard regression used only the variables that were significant in the univariate analysis. P-values less than 0.05 were considered statistically significant.

\section{Results}

RT-PCR analysis of KGFR in gastric cancer cell lines. To examine the expression patterns of KGFR in human cultured gastric cancer cell lines, RT-PCR was performed. A positive band at $150 \mathrm{bp}$ corresponding to KGFR mRNA was detected in the NUGC-4, KATO-III and MKN-7 cell lines, but not in the NS-8 cell line (Fig. 1, upper panel). B-actin mRNA was

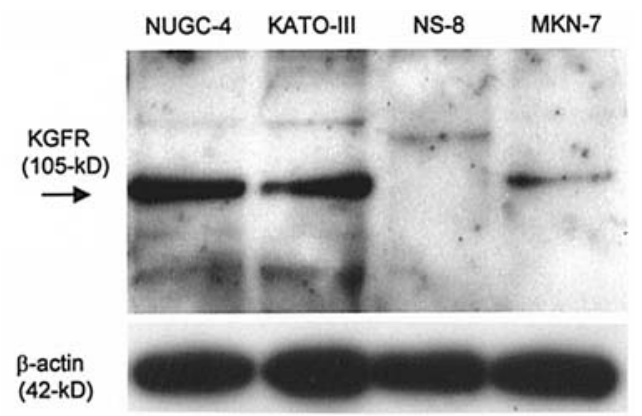

Figure 2. Westen blot analysis of KGFR protein in gastric cancer cell lines. The 105-kDa bands corresponding to KGFR protein were detected in NUGC-4, KATO-III and MKN-7 gastric cancer cell lines (upper panel). B-actin protein with a molecular weight of $42 \mathrm{kDa}$ was almost equally detected (lower panel).

almost equally detected in all cancer cell lines (Fig. 1, lower panel).

Western blot analysis of KGFR in gastric cancer cell lines. To characterize KGFR protein expression in gastric cancer cell lines, total protein was extracted from the NUGC-4, KATO-III, NS-8 and MKN-7 cell lines. Western blot analysis of these cell lines using the anti-KGFR antibody showed a single band corresponding to the KGFR protein at $105 \mathrm{kDa}$ in the NUGC-4, KATO-III and MKN-7 cell lines, but not in the NS-8 cell line (Fig. 2, upper panel). B-actin protein at $42 \mathrm{kDa}$ was almost equally detected in all four cancer cell lines (Fig. 2, lower panel).

Localization of KGFR and Ki-67 in non-cancerous gastric tissue. To examine the localization of the KGFR protein in human gastric cancer tissue, immunohistochemical staining was performed. In non-cancerous gastric tissue, KGFR immunoreactivity was weakly detected in the cell membrane and cytoplasm in the luminal surface of epithelial cells (Fig. 3A). KGFR was strongly expressed in the gastric pits and lesions of intestinal metaplasia (Fig. 3C and E, arrows). In contrast, Ki-67 immunoreactivity was detected in the nucleus of epithelial cells in the basal and parabasal regions (Fig. 3B, D and F, arrows). In addition, KGFR immunoreactivity was strongly detected in the nucleus and cytoplasm of parietal cells (Fig. 4A and B, arrows). To eliminate the possibility of non-specific staining of the nucleus of parietal cells with DAB, New Fuchsin was used as the substrate for HRP. KGFR was strongly detected in the nucleus and cytoplasm of the parietal cells with New Fuchsin staining (Fig. 4C, arrows). Other techniques, such as immunofluorescence staining and confocal laser microscopy, were performed to confirm the expression of KGFR in the nucleus of gastric parietal cells. Strong KGFR signals were homogeneously detected in the parietal cells (Fig. 4D, arrow). Furthermore, the nucleus that was stained with DAPI was detected in the same section, indicating the KGFR location in the nucleus of parietal cells (Fig. 4E and F, arrow).

Correlation of KGFR expression and clinicopathological factors in gastric cancer. In gastric cancer tissue, KGFR was localized in the cell membrane and cytoplasm of cancer cells and KGFR-positive cells were diffusely distributed in the 


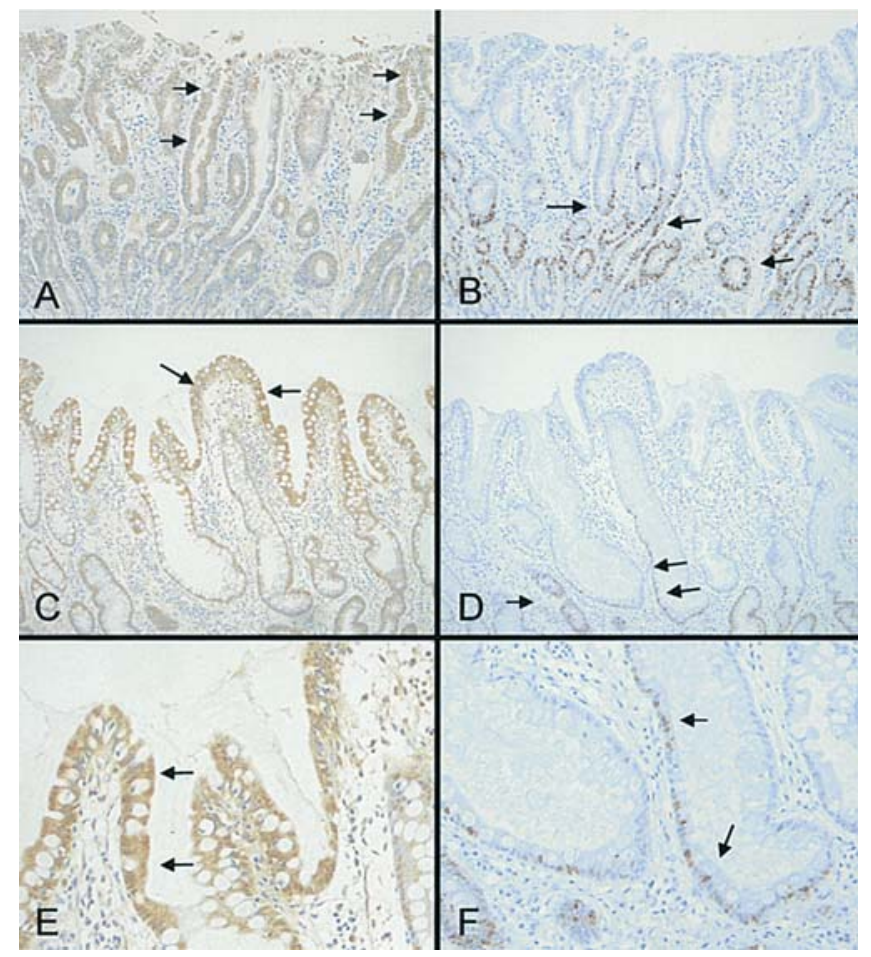

Figure 3. Immunohistochemical analysis of KGFR in non-cancerous gastric tissue. In non-cancerous gastric tissue, KGFR immunoreactivity was weakly detected in the cell membrane and cytoplasm in the luminal surface of gastric epithelial cells (A, arrows). In contrast, Ki-67 immunoreactivity was detected in the nucleus of epithelial cells in the basal and parabasal regions (B, D and F, arrows). KGFR was more strongly detected in the gastric epithelial cells of intestinal metaplastic lesions (C and E). A, C and E, KGFR immunohistochemistry; B, D and F, Ki-67 immunohistochemistry. Original magnification: A-D, x200; E and F, x400

cancer cell nests in serial sections (Fig. 5A, C and E, respectively). Ki-67 was localized in the nucleus of the cancer cells (Fig. 5B, D and F).

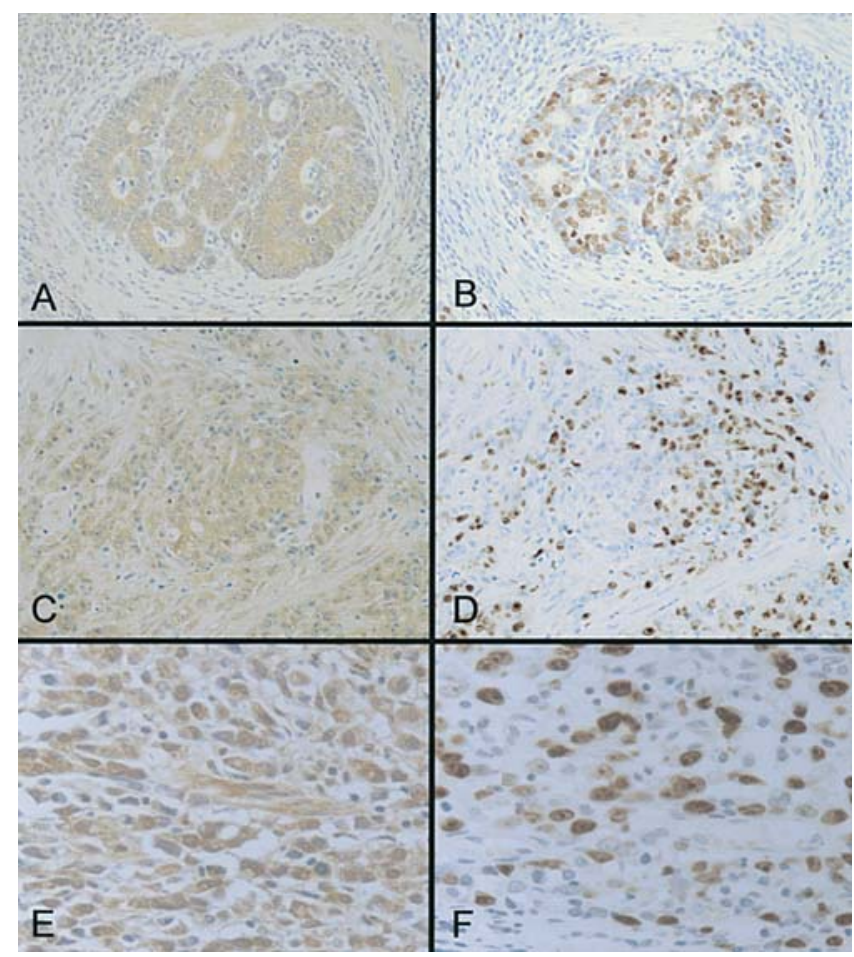

Figure 5. Immunohistochemical analyses of KGFR in gastric cancer tissue KGFR immunoreactivity was detected in the cytoplasm of gastric cancer cells, classified as well-differentiated adenocarcinoma (A), poorly differentiated adenocarcinoma (C) and signet-ring cell carcinoma (B). Ki-67 was localized in the nucleus of the gastric cancer cells in the serial sections (B, D and F). A, C and E, KGFR immunohistochemistry; B, D and F, Ki-67 immunohistochemistry. Original magnification: A-D, x200; E and F, x400.

KGFR was expressed in 46 of 126 (36.5\%) gastric cancer cases. The correlation between KGFR expression and clinicopathological factors is summarized in Table II. KGFR expression was significantly associated with early-type macroscopic findings, shallow invasion of the gastric wall,

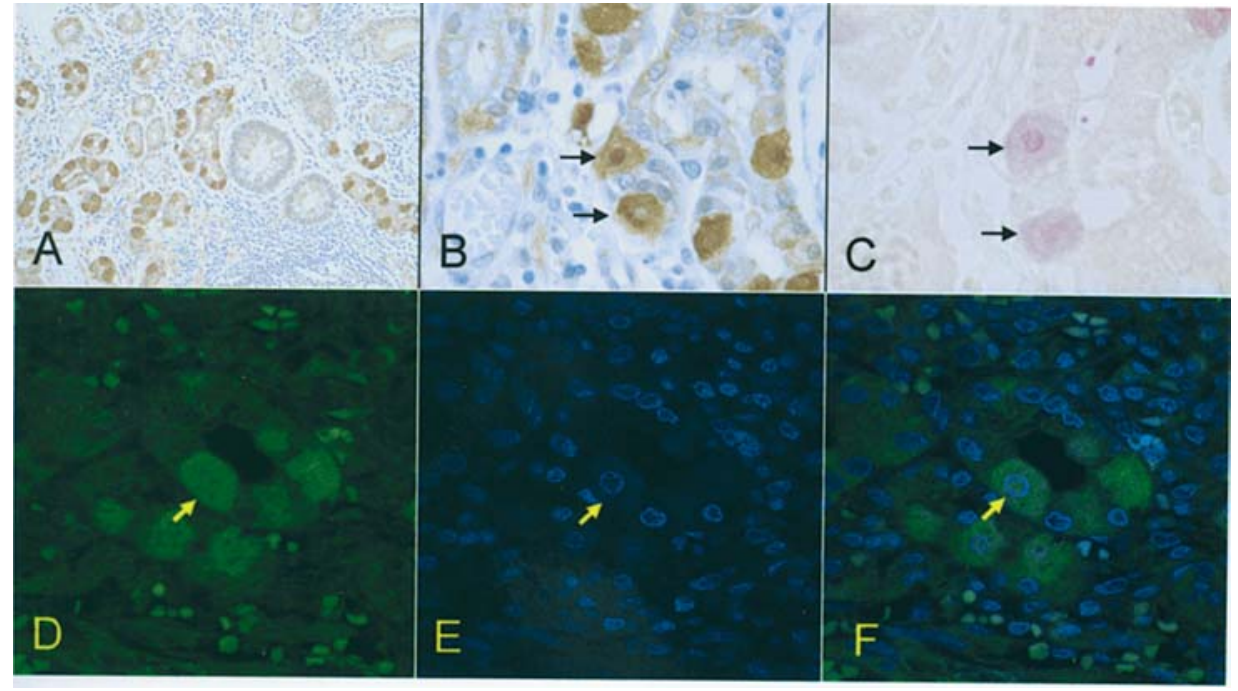

Figure 4. Immunohistochemical analysis of KGFR in parietal cells. KGFR immunoreactivity was weakly detected in the cytoplasm of many parietal cells and strongly detected in some nuclei of the cells (A and B, arrows). This KGFR expression in the nuclei of parietal cells was confirmed using New Fuchsin as the substrate (C, arrows). Strong KGFR signals were homogeneously detected in the parietal cells (D, arrow). The nucleus that was stained with DAPI was detected in the same section (E, arrow). An FITC image and a DAPI image are shown in the same section (F, arrow). Original magnification: A, x200; B and C, $x 400 ;$ D-F, x600 
Table II. Clinicopathological factors and expression of KGFR.

\begin{tabular}{|c|c|c|c|}
\hline \multirow[b]{2}{*}{ Variables } & \multicolumn{2}{|c|}{ Expression of KGFR } & \multirow[b]{2}{*}{ p-value } \\
\hline & Positive $(n=46)$ & Negative $(n=80)$ & \\
\hline Gender & & & NS \\
\hline Female & 16 & 30 & \\
\hline Male & 30 & 50 & \\
\hline Age (years) & & & NS \\
\hline$<60$ & 11 & 28 & \\
\hline$>61$ & 35 & 52 & \\
\hline Macroscopic findings & & & 0.0188 \\
\hline 0 & 26 & 28 & \\
\hline $1,2,3,4$ & 20 & 52 & \\
\hline Tumor size (cm) & & & NS \\
\hline$<2$ & 13 & 15 & \\
\hline$\geq 2$ & 33 & 65 & \\
\hline Histopathological type & & & NS \\
\hline Well-differentiated adenocarcinoma & 17 & 12 & \\
\hline Moderately differentiated adenocarcinoma & 7 & 22 & \\
\hline Poorly differentiated adenocarcinoma (solid) & 6 & 12 & \\
\hline poorly differentiated adenocarcinoma (non-solid) & 6 & 13 & \\
\hline Signet-ring cell carcinoma & 8 & 17 & \\
\hline Mucinous adenocarcinoma & 0 & 4 & \\
\hline Papillary adenocarcinoma & 2 & 0 & \\
\hline Depth & & & 0.0283 \\
\hline $\mathrm{m}$ & 16 & 14 & \\
\hline sm, mp, ss, se, si & 30 & 66 & \\
\hline Stromal reaction $^{\mathrm{a}}$ & & & NS \\
\hline Medullary type & 9 & 11 & \\
\hline Intermediate type & 14 & 33 & \\
\hline Scirrhous type & 9 & 20 & \\
\hline Growth pattern & & & 0.0119 \\
\hline Expansive type & 19 & 14 & \\
\hline Intermediate type & 18 & 48 & \\
\hline Infiltrative type & 9 & 18 & \\
\hline Lymphatic invasion & & & NS \\
\hline Negative & 15 & 19 & \\
\hline Positive & 31 & 61 & \\
\hline Venous invasion & & & NS \\
\hline Negative & 35 & 49 & \\
\hline Positive & 11 & 31 & \\
\hline Regional lymph node metastasis & & & NS \\
\hline Negative & 24 & 30 & \\
\hline Positive & 22 & 50 & \\
\hline Stage & & & 0.0149 \\
\hline I & 25 & 30 & \\
\hline II & 6 & 8 & \\
\hline III & 13 & 20 & \\
\hline IV & 2 & 22 & \\
\hline
\end{tabular}

NS, not significant. ${ }^{3} 30$ samples (depth m) were excluded, according to Japanese Classification of Gastric Carcinoma (25). 


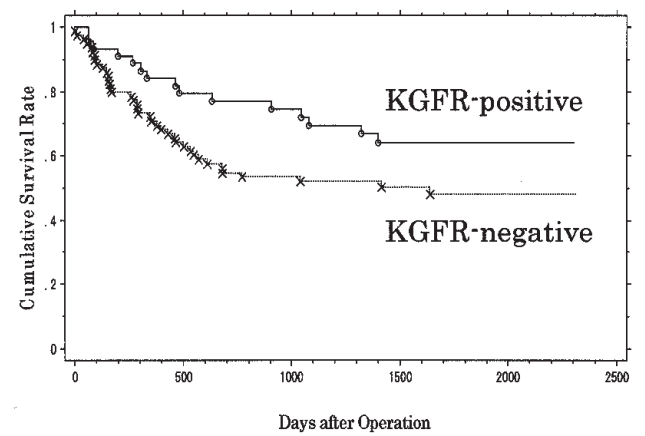

Figure 6. Cumulative survival curves of KGFR-positive and KGFR-negative patients. The overall survival rate of patients who were KGFR-positive was higher than that of patients who were KGFR-negative.

expansive growth type and the early stage of disease (Table II). No appreciable correlation was demonstrated between KGFR expression and either gender, age, tumor size, histopathological type, stromal reaction, lymphatic invasion, venous invasion or regional lymph node metastasis.

Correlation of KGFR expression and patient survival. The overall survival rate of all patients who were KGFR-positive was higher than that of patients who were KGFR-negative (Fig. 6). However, no statistically significant difference was observed between the two groups.

\section{Discussion}

In this study, we investigated the KGFR expression in four gastric cancer cell lines and detected it in all four cell lines except the NS-8 cell line. $K$-sam genes have been reported to be expressed in seven of eight gastric cancer cell lines but not in the MKN-1 cell line (20). MKN-1 cells were isolated from a metastatic lesion of an adenosquamous carcinoma of the liver. Although some researchers include $K$-sam genes in the category of Kgfr, Fgfr 2 and bek genes, it is considered that $K$-sam IIcl genes actually correspond to Kgfr genes (27). To our knowledge, this is the first report on KGFR protein expression in gastric cancer cell lines using a specific antibody against KGFR. NS-8 is a unique adenocarcinoma cell line that produces $\alpha$-fetoprotein (AFP). The chromosomal localizations of KGFR and AFP (10q26 and 4q11-22, respectively) are different, and the relationship between AFP and the lack of KGFR expression is not clear at present.

In non-cancerous gastric tissue, we detected KGFR-positive sites in the nucleus and cytoplasm of parietal cells in the fundus. The expression of KGFR protein in the nucleus of parietal cells was also confirmed by immunofluorescence staining and confocal laser microscopy. In general, intranuclear receptors are receptors for lipid-soluble ligands and include dioxin receptors and a group of intranuclear steroid receptors (28-31). The intranuclear steroid receptor group in humans includes 48 types of receptor, including those for thyroid hormone, steroid hormones such as estrogen, and lipid-soluble vitamins. These intranuclear steroid receptors are ligand-induced transcription inhibitors and control the expression of specific target genes at the transcription level in response to signals from lipid-soluble ligands. The dioxin receptor is the only known intranuclear receptor for the drug, dioxin, an endocrine disruptor. On the other hand, FGFRs including KGFR are reportedly present in the cell membrane and cytoplasm. The mechanism by which KGFR protein was expressed in the nuclei of gastric parietal cells is not clear. However, intranuclear expression of FGF-2 (basic-FGF) was previously reported, and FGF-1, FGF-2, FGF-3 and FGF-11FGF-14 were shown to possess nuclear location signals $(8,32)$. Parietal cells were reported to proliferate after the addition of recombinant FGF-7, and the proliferation decreases with the depletion of recombinant FGF-7 (13). These findings suggest that intranuclear KGFR has a specific function in parietal cells with ligands for KGFR.

The Ki-67 antigen is expressed in the nucleus of cells in all active phases of the cell cycle (G1, S, G2 and M phases) (33). In gastric cancer tissue, there was no significant difference between KGFR-positive and Ki-67-positive sites in the cancer cell nests. Previously, we reported that KGFR is localized at the center of cancer cell nests, whereas Ki-67 is localized in the periphery of colorectal cancer tissue $(34,35)$. Furthermore, similar KGFR and Ki-67 localizations were reported in cancer cell nests in pulmonary squamous cell carcinoma. In contrast, KGFR and Ki-67 were reported to be co-localized in lung adenocarcinoma (36). The localization of KGFR in gastric cancer tissue is similar to that of KGFR in lung adenocarcinoma. The higher expression rate of KGFR in welldifferentiated adenocarcinoma in the stomach is consistent with those in previous reports, including those observed in colorectal cancer and uterine cervical cancer $(35,37)$.

In this study, we investigated 126 patients and compared the clinicopathological factors between KGFR-positive and KGFR-negative patients. Significant differences were observed between the two groups in terms of macroscopic findings, extent of tumor invasion, growth pattern and clinical stage; for each, more remarkable in less-advanced cancer cases. Likewise, KGFR-positive sites were detected in the cytoplasm of more differentiated types of cancer cell, although no statistically significant difference was found. It was previously reported that more $K$-sam genes are expressed in poorlydifferentiated type IV gastric cancer cells and are associated with invasion, proliferation and metastasis (20). The difference between this finding and ours may be caused by the difference between the isoforms of $\mathrm{K}$-sam used, other than KGFR, as mentioned earlier. In addition, KGF is secreted by gastric fibroblasts and is involved in the proliferation of scirrhous gastric cancer. Moreover, the administration of recombinant KGF induced more proliferation in scirrhous gastric cancer (24). These suggest the importance of researching the expression of KGFR ligands, including FGF-1, FGF-3, FGF4, FGF-6, FGF-7 and FGF-10 in gastric cancer tissue.

In conclusion, KGFR was localized in the luminal surfaces of normal gastric epithelial cells and in the nuclei of parietal cells. The KGFR protein was more frequently detected during expansive growth and in the early stages of gastric cancer. These lines of evidence suggest that KGFR expression correlates with the differentiation of normal gastric epithelial cells and parietal cell functions. The decreased expression level of KGFR in advanced stages of gastric cancer may be associated with the proliferation and invasion of gastric cancer and a poor prognosis for patients. 


\section{Acknowledgments}

We thank Ms. Y. Nagasawa (Division of Pathology, Second Hospital of Nippon Medical School) for preparing the tissue sections, and Ms. K. Kawahara and Mr. K. Teduka, (Department of Pathology II, Nippon Medical School) for their excellent technical assistance. This work was supported in part by Grants-in-Aid for Scientific Research (nos. 16591365 and 16591360) from the Japan Society for the Promotion of Science, and a Grant-in-Aid for Young Scientists (no. 16790765) from The Ministry of Education, Culture, Sports, Science and Technology.

\section{References}

1. Yoshida K, Yasui W, Ito H and Tahara E: Growth factors in progression of human esophageal and gastric carcinomas. Exp Pathol 40: 291-300, 1990.

2. Tahara E: Growth factors and oncogenes in human gastrointestinal carcinomas. J Cancer Res Clin Oncol 116: 121-131, 1990

3. Tokunaga A, Onda M, Okuda T, Teramoto T, Fujita I, Mizutani T, Kiyama T, Yoshiyuki T, Nishi K and Matsukura N: Clinical significance of epidermal growth factor (EGF), EGF receptor, and c-erbB-2 in human gastric cancer. Cancer 75: 1418-1425, 1995.

4. Minchiotti G, Parisi S, Liguori GL, D'Andrea D and Persico MG: Role of the EGF-CFC gene cripto in cell differentiation and embryo development. Gene 287: 33-37, 2002.

5. Ornitz DM and Itoh N: Fibroblast growth factors. Genome Biol 2: Reviews 3005, 2001.

6. Itoh N and Ornitz DM: Evolution of the Fgf and Fgfr gene families. Trends Genet 20: 563-569, 2004.

7. Miki T, Bottaro DP, Fleming TP, Smith CL, Burgess WH, Chan AM and Aaronson SA: Determination of ligand-binding specificity by alternative splicing: two distinct growth factor receptors encoded by a single gene. Proc Natl Acad Sci USA 89: 246-250, 1992

8. Mohammadi M, Olsen SK and Ibrahimi OA: Structural basis for fibroblast growth factor receptor activation. Cytokine Growth Factor Rev 16: 107-137, 2005.

9. Igarashi M, Finch PW and Aaronson SA: Characterization of recombinant human fibroblast growth factor (FGF)-10 reveals functional similarities with keratinocyte growth factor (FGF-7). J Biol Chem 273: 13230-13235, 1998.

10. Xu X, Weinstein M, Li C, Naski M, Cohen RI, Ornitz DM, Leder P and Deng C: Fibroblast growth factor receptor 2 (FGFR2)-mediated reciprocal regulation loop between FGF8 and FGF10 is essential for limb induction. Development 125: 753-765, 1998.

11. De Moerlooze L, Spencer-Dene B, Revest J, Hajihosseini M, Rosewell I and Dickson C: An important role for the IIIb isoform of fibroblast growth factor receptor 2 (FGFR2) in mesenchymal-epithelial signalling during mouse organogenesis. Development 127: 483-492, 2000.

12. Revest JM, Spencer-Dene B, Kerr K, De Moerlooze L, Rosewell I and Dickson C: Fibroblast growth factor receptor 2-IIIb acts upstream of Shh and Fgf4 and is required for limb bud maintenance but not for the induction of Fgf8, Fgf10, Msx1, or Bmp4. Dev Biol 231: 47-62, 2001.

13. Housley RM, Morris CF, Boyle W, Ring B, Biltz R, Tarpley JE, Aukerman SL, Devine PL, Whitehead RH and Pierce GF: Keratinocyte growth factor induces proliferation of hepatocytes and epithelial cells throughout the rat gastrointestinal tract. J Clin Invest 94: 1764-1777, 1994.

14. Visco V, Belleudi F, Marchese C, Leone L, Aimati L, Cardinali G, Kovacs D, Frati L and Torrisi MR: Differential response to keratinocyte growth factor receptor and epidermal growth factor receptor ligands of proliferating and differentiating intestinal epithelial cells. J Cell Physiol 200: 31-44, 2004.

15. Otte JM, Schmitz F, Banasiewicz T, Drews M, Flolsch UR and Herzig KH: Expression of keratinocyte growth factor and its receptor in colorectal cancer. Eur J Clin Invest 30: 222-229, 2000.

16. Ishiwata T, Friess H, Buchler MW, Lopez ME and Korc M: Characterization of keratinocyte growth factor and receptor expression in human pancreatic cancer. Am J Pathol 153: 213-222, 1998
17. Siddiqi I, Funatomi H, Kobrin MS, Friess H, Buchler MW and Korc M: Increased expression of keratinocyte growth factor in human pancreatic cancer. Biochem Biophys Res Commun 215: 309-315, 1995.

18. Dignass AU, Tsunekawa S and Podolsky DK: Fibroblast growth factors modulate intestinal epithelial cell growth and migration. Gastroenterology 106: 1254-1262, 1994.

19. Zhang Y, Wang H, Toratani S, Sato JD, Kan M, McKeehan WL and Okamoto T: Growth inhibition by keratinocyte growth factor receptor of human salivary adenocarcinoma cells through induction of differentiation and apoptosis. Proc Natl Acad Sci USA 98: 11336-11340, 2001

20. Hattori Y, Itoh H, Uchino S, Hosokawa K, Ochiai A, Ino Y, Ishii H, Sakamoto H, Yamaguchi N, Yanagihara K, Hirohashi S, Sugimura $\mathrm{T}$ and Terada M: Immunohistochemical detection of K-sam protein in stomach cancer. Clin Cancer Res 2: 1373-1381, 1996.

21. Iida S, Katoh O, Tokunaga A and Terada M: Expression of fibroblast growth factor gene family and its receptor gene family in the human upper gastrointestinal tract. Biochem Biophys Res Commun 199: 1113-1119, 1994.

22. Yashiro M, Chung YS and Sowa M: Role of orthotopic fibroblasts in the development of scirrhous gastric carcinoma. Jpn J Cancer Res 85: 883-886, 1994.

23. Yashiro M, Chung YS, Kubo T, Hato F and Sowa M: Differential responses of scirrhous and well-differentiated gastric cancer cells to orthotopic fibroblast. Br J Cancer 74: 1096-1103, 1996.

24. Nakazawa K, Yashiro M and Hirakawa K: Keratinocyte growth factor produced by gastric fibroblasts specifically stimulates proliferation of cancer cells from scirrhous gastric carcinoma. Cancer Res 63: 8848-8852, 2003.

25. Japan Research Society for Gastric Cancer: Japanese Classification of Gastric Carcinoma. Kanehara, Tokyo, 1999.

26. Ishiwata T, Naito Z, Lu YP, Kawahara K, Fujii T, Kawamoto Y, Teduka $\mathrm{K}$ and Sugisaki Y: Differential distribution of fibroblast growth factor (FGF)-7 and FGF-10 in L-arginine-induced acute pancreatitis. Exp Mol Pathol 73: 181-190, 2002.

27. Ishii H, Yoshida T, Oh H, Yoshida S and Terada M: A truncated $\mathrm{K}$-sam product lacking the distal carboxyl-terminal portion provides a reduced level of autophosphorylation and greater resistance against induction of differentiation. Mol Cell Biol 15: 3664-3671, 1995.

28. Belandia B and Parker MG: Nuclear receptors: a rendezvous for chromatin remodeling factors. Cell 114: 277-280, 2003.

29. Mangelsdorf DJ, Thummel C, Beato M, Herrlich P, Schutz G, Umesono K, Blumberg B, Kastner P, Mark M, Chambon P and Evans M: The nuclear receptor superfamily; the second decade. Cell 83: 835-839, 1995.

30. Makishima M, Lu TT, Xie W, Whitfield GK, Demot H, Evans RM, Haussler MR and Mangelsdorf DJ: Vitamin D receptor as an intestinal bile acid sensor. Science 296: 1313-1316, 2003.

31. Makoto M: Nuclear receptors as targets for drug development: regulation of cholesterol and bile acid metabolism by nuclear receptor. J Pharmacol Sci 97: 117-183, 2005.

32. Arese M, Chen Y, Florkiewicz RZ, Gualandris A, Shen B and Rifkin DB: Nuclear activities of basic fibroblast growth factor: potentiation of low-serum growth mediated by natural or chimeric nuclear localization signals. Mol Biol Cell 10: 1429-1444, 1999.

33. Scholzen T and Gerdes J: The Ki-67 protein: from the known and the unknown. J Cell Physiol 182: 311-322, 2000.

34. Watanabe M, Ishiwata T, Nishigai K, Moriyama Y and Asano G: Overexpression of keratinocyte growth factor in cancer cells and enterochromaffin cell in human colorectal cancer. Pathol Int 50: 363-372, 2000.

35. Yoshino M, Ishiwata T, Watanabe M, Komine O, Shibuya T, Tokunaga A and Naito Z: Keratinocyte growth factor receptor expression in normal colorectal epithelial cells and differentiated type of colorectal cancer. Oncol Rep 13: 247-252, 2005.

36. Yamayoshi T, Nagayasu T, Matsumoto K, Abo T, Hishikawa Y and Koji T: Expression of keratinocyte growth factor/fibroblast growth factor-7 and its receptor: correlation with tumour proliferative activity and patient prognosis. J Pathol 204: 110-118, 2004.

37. Kurban G, Ishiwata T, Kudo M, Yokoyama M, Sugisaki Y and Naito Z: Expression of keratinocyte growth factor receptor (KGFR/FGFR2IIIb) in human uterine cervical cancer. Oncol Rep 11: 987-991, 2004. 